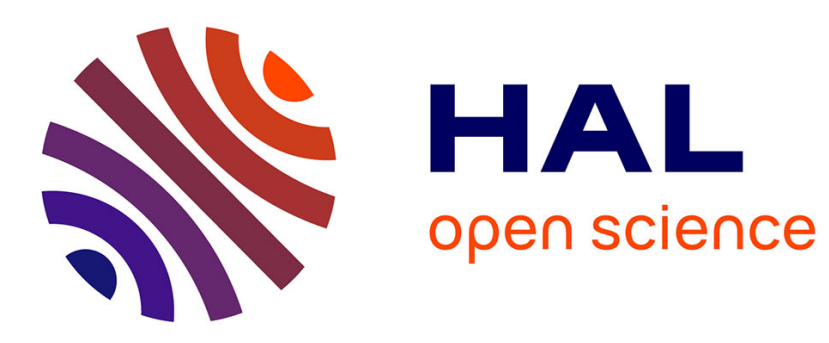

\title{
Imagerie médicale computationnelle (radiomique) et potentiel en immuno-oncologie
}

Roger Sun, Elaine Johanna Limkin, Laurent Dercle, Sylvain Reuzé, Evangelia

I Zacharaki, Cyrus Chargari, Antoine Schernberg, Anne-Sophie Dirand, Anthony Alexis, Nikos Paragios, et al.

\section{To cite this version:}

Roger Sun, Elaine Johanna Limkin, Laurent Dercle, Sylvain Reuzé, Evangelia I Zacharaki, et al.. Imagerie médicale computationnelle (radiomique) et potentiel en immuno-oncologie. Cancer/Radiothérapie, 2017, 21 (6-7), pp.648-654. 10.1016/j.canrad.2017.07.035 . hal-01668902

\section{HAL Id: hal-01668902 https://hal.inria.fr/hal-01668902}

Submitted on 20 Dec 2017

HAL is a multi-disciplinary open access archive for the deposit and dissemination of scientific research documents, whether they are published or not. The documents may come from teaching and research institutions in France or abroad, or from public or private research centers.
L'archive ouverte pluridisciplinaire HAL, est destinée au dépôt et à la diffusion de documents scientifiques de niveau recherche, publiés ou non, émanant des établissements d'enseignement et de recherche français ou étrangers, des laboratoires publics ou privés. 
Radiothérapie

Elsevier Editorial System(tm) for Cancer /

Manuscript Draft

Manuscript Number:

Title: Imagerie médicale computationnelle (radiomique) et potentiel en immuno-oncologie

Article Type: Mise au point/Review article

Section/Category: Submission for the SFRO's annual meeting / Article pour le congrès de la S.F.R.O.

Keywords: Radiomique, immunologie, oncologie, imagerie médicale computationnelle

Corresponding Author: Dr. Roger Sun,

Corresponding Author's Institution: Gustave Roussy

First Author: Roger Sun

Order of Authors: Roger Sun; Elaine Johanna Limkin; Laurent Dercle; Sylvain Reuzé; Evangelia I Zacharaki; Cyrus Chargari; Antoine Schernberg; Anne-Sophie Dirand; Anthony Alexis; Nikos Paragios; Eric Deutsch; Charles Ferté; Charlotte Robert

Abstract: L'arrivée de l'immunothérapie a profondément modifié la prise en charge de multiples cancers, permettant des réponses tumorales jusqu'alors inespérées, même si une majorité des patients ne répondent pas à ces nouveaux traitements. L'identification de biomarqueurs permettant de cibler les patients répondeurs est un enjeu majeur. L'imagerie médicale computationnelle (ou radiomique) est une discipline récente et extrêmement prometteuse. Elle consiste en l'analyse informatique d'images médicales et les traduit en données quantitatives complexes. Ces données de haute-dimension permettent une caractérisation et une analyse plus en profondeur du phénotype tumoral. L'imagerie médicale computationnelle présente l'avantage d'être non-invasive, de pouvoir évaluer la maladie tumorale dans sa globalité, et de pouvoir être répétée dans le temps pour suivre l'évolution tumorale au cours du temps. L'imagerie médicale computationnelle a pour objectif final de déterminer des biomarqueurs d'imagerie apportant une aide à la décision médicale et permettant aussi de mieux comprendre la biologie du cancer. Cette revue développera le processus de l'analyse en imagerie computationnelle, et présentera le potentiel de son utilisation en immuno-oncologie. 
Mise au point

\section{Imagerie médicale computationnelle (radiomique) et potentiel en immuno-oncologie}

\section{Computational medical imaging (radiomics) and potential for immuno-oncology}

R. Sun ${ }^{1,2}$, E.J. Limkin ${ }^{1,2}$, L. Dercle ${ }^{4,5}$, S. Reuzé ${ }^{1,3}$, E.I. Zacharaki ${ }^{6}$, C. Chargari ${ }^{1,2,7,8}$, A. Schernberg $^{1,2}$, A.S. Dirand ${ }^{1}$, A. Alexis ${ }^{1}$, N. Paragios ${ }^{5,8}$, E. Deutsch ${ }^{1,2,10}$, C. Ferté $^{1,11}$, C. Robert $^{1,2,3,10}$

${ }^{1}$ Radiomics team, INSERM U1030, Gustave Roussy, Université Paris-Sud Paris-Saclay, F94805, Villejuif, France

2 Département de Radiothérapie, Université Paris-Sud Paris-Saclay, F-94805, Villejuif, France

${ }^{3}$ Département de Physique médicale, Université Paris-Sud Paris-Saclay, F-94805, Villejuif, France

${ }^{4}$ Département de médecine nucléaire, Gustave Roussy, Université Paris-Sud Paris-Saclay, F94805, Villejuif, France

${ }^{5}$ INSERM U1015, F-94805, Villejuif France

${ }^{6}$ TheraPanacea, Paris, 75014, France

${ }^{7}$ Institut de Recherche Biomédicale des Armées, D19, 91220 Brétigny sur Orge, France

${ }^{8}$ Service de santé des armées, Ecole du Val-de-Grace, 75005, Paris, France.

${ }^{9}$ Centre de Vision Numérique, CentraleSupelec/ Université Paris-Sud Paris-Saclay /Inria, 92295, Châtenay-Malabry, France

${ }^{10}$ Faculté de Médecine, Université Paris-Sud Paris-Saclay, 94270, Kremlin-Bicêtre, France

${ }^{11}$ Département de cancérologie ORL, Université Paris-Sud Paris-Saclay, F-94805, Villejuif, France

* Lauréat de la bourse Maurice Tubiana 2016

Auteur Correspondant :

Roger SUN

INSERM U1030

Gustave Roussy

94805 Villejuif Cedex

France

Tel +33(0)142115050

E-mail : roger.sun@gustaveroussy.fr

Conflits d'intérêts: aucun

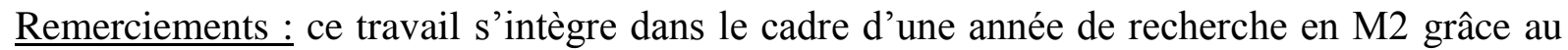
soutien financier de la SFRO, du SNRO et de la SFjRO via la bourse Maurice Tubiana.

Titre courant : Imagerie médicale computationnelle et immuno-oncologie 


\section{Résumé :}

L'arrivée de l'immunothérapie a profondément modifié la prise en charge de multiples cancers, permettant des réponses tumorales jusqu'alors inespérées, même si une majorité des patients ne répondent pas à ces nouveaux traitements. L'identification de biomarqueurs permettant de cibler les patients répondeurs est un enjeu majeur.

L'imagerie médicale computationnelle (ou radiomique) est une discipline récente et extrêmement prometteuse. Elle consiste en l'analyse informatique d'images médicales et les traduit en données quantitatives complexes. Ces données de haute-dimension permettent une caractérisation et une analyse plus en profondeur du phénotype tumoral. L'imagerie médicale computationnelle présente l'avantage d'être non-invasive, de pouvoir évaluer la maladie tumorale dans sa globalité, et de pouvoir être répétée dans le temps pour suivre l'évolution tumorale au cours du temps. L'imagerie médicale computationnelle a pour objectif final de déterminer des biomarqueurs d'imagerie apportant une aide à la décision médicale et permettant aussi de mieux comprendre la biologie du cancer. Cette revue développera le processus de l'analyse en imagerie computationnelle, et présentera le potentiel de son utilisation en immuno-oncologie.

Mots clefs :

Radiomique, immunologie, oncologie, imagerie médicale computationnelle 
Abstract:

The arrival of immunotherapy has profoundly changed the management of multiple cancers, obtaining unexpected tumor responses. However, until now, majority of patients do not respond to these new treatments. The identification of biomarkers to determine precociously responding patients is a major challenge.

Computational medical imaging (also known as radiomics) is a promising and rapidly growing discipline. This new approach consists in the analysis of high dimensional data extracted from medical imaging, to further describe tumor phenotypes. This approach has the advantages of being non-invasive, being capable of evaluating the tumor and its microenvironment in their entirety, thus characterizing spatial heterogeneity, and being easily repeatable over time. The end goal of radiomics is to determine imaging biomarkers as decision support tools for clinical practice and to facilitate better understanding of cancer biology, allowing the assessment of the changes throughout the evolution of the disease and the therapeutic sequence. This review will develop the process of computational imaging analysis and present its potential in immuno-oncology.

Key words:

Radiomics, immunology, oncology, computational medical imaging 


\section{Introduction}

L'imagerie médicale computationnelle (ou radiomique) est une discipline récente et extrêmement prometteuse [1]. Alors que l'analyse classique des examens d'imagerie standard tels que les scanners, IRM (Imagerie par Résonance Magnétique) ou TEP (Tomographie par Emission de Positons) repose sur l'interprétation visuelle de caractéristiques simples - comme la taille de la tumeur, sa forme générale, la prise de contraste ou l'intensité du signal - cette nouvelle approche traite informatiquement ces examens d'imagerie et les traduit en données quantitatives complexes. Ces données de haute-dimension permettent une caractérisation et une analyse plus en profondeur du phénotype tumoral [1-3]. L'hypothèse sous-jacente est que les données issues de ces examens reflètent l'architecture des tissus, mais aussi leur composition cellulaire et moléculaire. L'imagerie médicale computationnelle a pour objectif final de développer des biomarqueurs d'imagerie apportant une aide à la décision médicale et permettant aussi de mieux comprendre la biologie du cancer [4-6]. Cette approche présente plusieurs avantages: (i) elle est non-invasive, (ii) elle évalue la tumeur et son microenvironnement dans sa globalité, caractérisant ainsi son hétérogénéité spatiale, et (iii) peut être répétée dans le temps, permettant d'appréhender les modifications tout au long de l'histoire de la maladie et de la séquence thérapeutique.

L'arrivée de l'immunothérapie a profondément modifié la prise en charge de multiples cancers tels que les mélanomes [7,8], les lymphomes [9], les cancers pulmonaires [10], et rénaux [11], avec des résultats jusqu'alors inespérés : de 10 à $40 \%$ de réponses tumorales objectives tous types confondus, et jusqu'à $60 \%$ dans les mélanomes chez des patients aux stades extrêmement avancés [12-14]. L'identification de biomarqueurs permettant de cibler les patients susceptibles de répondre à ces traitements est un enjeu majeur. Un des axes de 
recherche actuel est l'utilisation de l'imagerie médicale computationnelle comme prédicteur de l'infiltration tumorale lymphocytaire et ainsi de l'efficacité des immunothérapies.

L'objectif de cette revue est de présenter dans un premier temps les différentes étapes du processus de l'imagerie médicale computationnelle, puis, dans un second temps, les potentiels de son utilisation en immuno-oncologie.

\section{Processus de l'analyse en imagerie médicale computationnelle}

Le processus de l'analyse en imagerie médicale computationnelle consiste en plusieurs étapes distinctes : acquisition et segmentation d'images, extraction des caractéristiques, apprentissage statistique (Figure 1).

\section{Acquisition et segmentation d'images}

L'imagerie médicale computationnelle analyse de façon quantitative la répartition des intensités du signal dans une région ou un volume d'intérêt (ROI / VOI), et peut s'appliquer à des modalités d'imageries différentes et/ou multiples, avec pour chacune des intérêts et des limites qu'il est nécessaire de prendre en compte. Tout d'abord, la résolution spatiale des images varie selon les modalités. Elle est égale à environ $1 \mathrm{~mm}$ pour le scanner et l'IRM, et 5 mm pour le TEP. De ce fait, les ROI trop petites (nodules infra-centimétriques par exemple) peuvent ne pas fournir d'informations suffisantes pour une analyse computationnelle, alors que les ROI trop volumineuses peuvent être affectées par l'hétérogénéité des tumeurs (comme dans le cadre de tumeurs avec un centre nécrotique). Les mouvements du patient et de sa respiration pendant l'acquisition affectent également la qualité des images reconstruites, particulièrement sur un examen TEP qui dure plusieurs minutes, alors qu'un examen scanner est généralement acquis en quelques secondes. Ainsi, pour les premiers, l'acquisition 
représente un signal moyenné sur l'ensemble des positions de la tumeur, contre un seul temps

Plusieurs méthodes peuvent être utilisées pour définir les ROI à analyser. Les méthodes de segmentation automatique sont préférables pour la précision et la reproductibilité. Il a été prouvé que les variabilités inter et intra-observateurs sont plus faibles grâce aux outils de segmentation automatique par rapport aux méthodes de délinéation semi-automatique ou manuelle [17-20]. Cependant, le recours à la délinéation semi-automatique est le plus souvent nécessaire, car les méthodes automatisées ne sont utilisables que s'il existe de fortes différences de signal entre la lésion et le fond (tumeurs pulmonaires en CT et PET par exemple, et éventuellement dans certaines séquences d'IRM). Ainsi, en particulier pour les tumeurs situées au sein de structures saines relativement homogènes, la relecture par un médecin expérimenté est nécessaire pour la correction manuelle des contours prédéfinis par ordinateur. La reproductibilité de la segmentation est importante afin de diminuer une source de variabilité des caractéristiques radiomiques extraites, bien qu'il soit surtout important que ces caractéristiques extraites ne soient pas dépendantes de la variabilité à la segmentation [21]. 
Il existe plusieurs plateformes libres permettant de réaliser des contours automatiques et semiautomatiques, comme 3DSlicer (algorithme Growcut) [22]. Les contours générés peuvent être sauvés sous différents formats pour leur analyse. Le DICOM-RTSTRUCT est un format simple et courant pour le transfert des données en radiothérapie, et contient des informations sur les images et les ROI [23]. D'autres formats tels que Analyze [24] et NIfTI sont également utilisés [25].

\section{Extraction des caractéristiques d'imagerie (ou index)}

\section{Pré-traitement des images}

Les données brutes d'imagerie nécessitent un traitement préalable pour diminuer l'effet du bruit. Une étape facultative consiste à filtrer le signal contenu dans la ROI pour obtenir des images caractérisées par des résolutions spatiales différentes (lissage des images par filtres gaussiens par exemple), potentiellement utiles pour des analyses ultérieures [26]. Une autre étape consiste en la discrétisation ou le ré-échantillonnage des intensités du signal pour le calcul des index de second ordre. Il s'agit de diviser les valeurs continues potentiellement affectées aux voxels en plusieurs intervalles appelés «bins $»[27,28]$. Cette discrétisation peut être soit absolue (taille de bins fixe - par exemple 10 unités-Hounsfield (UH) pour un scanner), soit relative (nombre de bins fixe pour une même étude mais dont la taille varie en fonction des valeurs minimale et maximale prises par la tumeur). Le choix de la méthode est crucial car les index extraits varient en conséquence [21,29]. Plusieurs études ont montré qu'une discrétisation absolue permet d'extraire des index plus reproductibles et avait un potentiel discriminant plus important pour la caractérisation des tissus, avec l'avantage de ne pas dépendre du volume $[27,28,30]$.

Caractéristiques d'imagerie computationnelle (ou index radiomiques) 
Les caractéristiques sont classiquement divisées en plusieurs catégories: 1) les index quantitatifs de forme, relatifs à la taille, la forme, le volume et la surface de la tumeur, 2) les index de premier ordre (ou index d'histogramme), étudiant la distribution des valeurs des voxels sans considérer les relations spatiales (ex : moyenne, médiane, minimum, maximum, kurtosis, asymétrie, entropie...) [31], 3) les index de second ordre (ou index de texture), caractérisant les relations spatiales entre les voxels, principalement calculés à partir des matrices décrites par Haralick [32] (ex : matrice de co-occurrence [gray-level co-occurrence matrix, GLCM], matrice des longueurs des séries homogènes (gray-level run length matrix, GLRLM) [33], matrice des longueurs des zones homogènes (gray-level size zone matrix, GLZLM) [34], matrice de différence de niveaux de gris (neighborhood gray-level different matrix, NGLDM) [35]. Des transformées de Fourier et des filtres linéaires comme le filtre de Gabor peuvent être utilisés à la fois dans l'étape de prétraitement et pour l'extraction de caractéristiques spatiales ou fréquentielles [36,37]. Les caractéristiques extraites peuvent être globales (une valeur pour l'ensemble de la ROI) ou locales (une valeur par parcelle de la zone tumorale).

\section{Apprentissage statistique}

Implications de la haute dimension des variables radiomiques

L'imagerie médicale computationnelle intègre actuellement classiquement environ 50 à 500 caractéristiques quantitatives par patient [2,38], et certaines études vont jusqu'à 5000 caractéristiques [39]. Leur nombre devrait encore augmenter, alors que le nombre de patients analysés est souvent largement inférieur. Cette situation de "malédiction de la dimension", classique dans le domaine des omics (génomique, protéomique...), conduit à une probabilité forte d'obtenir des résultats faussement positifs [40-42]. Les ajustements pour les comparaisons multiples (correction de Bonferroni [43]) et le contrôle du taux de fausses 
découvertes (Benjamini-Hochberg [44]) sont des méthodes couramment utilisées pour en tenir compte. Le nombre important de variables entraîne aussi un risque de sur-apprentissage, se traduisant par un modèle parfait sur les données d'apprentissage mais sans aucune capacité de généralisation sur d'autres jeux de données. Ce risque peut être estimé par des méthodes de validation croisée pour l'apprentissage, et contrôlé par les méthodes de réduction de dimension [45]. A noter que l'utilisation de bases de données test-retest, où deux acquisitions par patient sont réalisées à quelques minutes d'intervalle, peut permettre d'éliminer dans un premier temps les index qui se révèlent peu robustes dans le temps [18,46].

\section{Méthodes de réduction de dimension}

L'éventail des différentes méthodes est large mais peut être classé en deux grandes catégories : la sélection de variables et l'extraction de caractéristiques construites à partir des variables initiales.

La première catégorie ne conserve qu'un sous-ensemble de variables, classées selon un critère de performance, éliminant ainsi les variables non-pertinentes et redondantes. La sélection des variables peut être effectuée selon 3 approches :

a) Approches «filtres » : la sélection des variables est réalisée dans une première étape de pré-traitement des données, indépendamment de la méthode d'apprentissage envisagée (par exemple, minimum Redundance Maximum Relevance [mRMR] [47], ReliefF [48]).

b) Approche «wrapper »: la sélection des variables est aussi réalisée dans une première étape de pré-traitement des données, mais le score de performance des variables est optimisé pour l'algorithme d'apprentissage spécifiquement envisagé qui va par exemple chercher un compromis entre le nombre de variables éliminées et le taux de réussite de classification sur un sous ensemble test. Ainsi les variables sont 


\section{Apprentissage statistique supervisé et non supervisé}

Les approches d'apprentissage statistiques les plus fréquentes sont les méthodes d'apprentissage supervisées. L'algorithme cherche à prédire une variable explicite dite variable de sortie (variable catégorielle pour les tâches de classification : bénin vs. malin, ou continue pour les tâches de régression : survie par exemple) à partir de variables d'entrées. Il est entrainé sur un premier jeu de données (training set), et évalué sur un second (validation set) qui doit être indépendant du premier [55]. Des méthodes de partition du jeu de données initial ou de validation croisées sont souvent utilisées lorsqu'une cohorte indépendante n'est pas disponible, mais l'interprétation des résultats doit être très prudente [56]. Les modèles de régression [45], les forêts aléatoires [57-62], les machines à vecteurs de support - support vector machine (SVM) [45,63,64] et plus récemment les réseaux de neurones profonds [65] semblent prometteurs dans le domaine de l'imagerie médicale computationnelle. 
Les méthodes non supervisées n'utilisent pas de variable de sortie prédéfinie. Brièvement,

leur objectif est de modéliser la structure sous-jacente des données pour en apprendre plus d'information. Il n'y a pas de «bonne réponse » sur laquelle entrainer le modèle. Ce sont les méthodes de partition de données - clustering, et les méthodes d'apprentissage des règles d'associations [66].

\section{Focus sur l'apprentissage profond-deep learning}

L'apprentissage profond fait référence à des méthodes avancées d'apprentissage statistique organisées en plusieurs couches, pour extraire des représentations des données sur de multiples niveaux, et dont les couches ne sont pas prédéfinies par l'utilisateur mais apprises directement depuis les données par l'algorithme, mimant ainsi le fonctionnement neuronal humain [67]. Ces méthodes récentes se sont montrées prometteuses dans chacune des étapes de l'apprentissage précédemment décrites.

Dans le domaine de l'imagerie médicale computationnelle, des méthodes de réseaux de neurones convolutifs profonds (CNN) [68] ou d'auto-encodeurs [69] se sont révélées performantes pour l'apprentissage hiérarchique non supervisé de caractéristiques d'imagerie de plus en plus complexes directement à partir de données brutes de l'image, permettant ainsi de « découvrir » les caractéristiques pertinentes, au lieu d'extraire des caractéristiques définies a priori par l'utilisateur. Une sélection de variables peut être réalisée de façon intégrée avec l'apprentissage des caractéristiques, et ce, à la fois sur les données brutes et sur les caractéristiques apprises [68-70]. De même, la classification supervisée peut aussi s'intégrer dans une même architecture avec les deux précédentes étapes pour optimiser et automatiser le processus [69,71]. Des études ont comparé les méthodes d'imagerie computationnelle «classiques » multi-étapes avec les méthodes d'apprentissage profond, et ont montré un meilleur pouvoir de classification et de prédiction de la mortalité avec les méthodes 
d'apprentissage profond dans le cas de nodules pulmonaires [71] ou de mammographies de dépistage du cancer du sein [69].

\section{Potentiel en immuno-oncologie}

La place de l'immuno-édition parmi les processus clés de l'oncogénèse est maintenant bien établie [72]. L'identification de signaux d'inactivation des lymphocytes T effecteurs comme PD-L1 (programmed death-ligand 1) a permis le développement de nouveaux traitements d'immunothérapie, et en particulier les anti PD-1/ PD-L1, révolutionnant le monde de l'oncologie [73-75]. Malgré l'existence de réponses spectaculaires et de longs répondeurs chez des patients en stade très avancés, celles-ci ne représentent que 10 à $40 \%$ des patients, soulignant la nécessité d'obtenir de nouveaux biomarqueurs [12,76]. Par ailleurs, plusieurs études cliniques ont montré que le profil de l'infiltration immunitaire tumorale et péritumorale préexistante est corrélé avec la réponse du patient à ces traitements [12,77-80]. En particulier, le phénotype immun-inflammatoire, caractérisé par la présence de cellules T-CD4 et CD8 dans le parenchyme tumoral est associée à une meilleure réponse clinique [77,81,82], que ce soit dans les mélanomes [83], les cancers ovariens [84], les cancers colorectaux [85], ou ceux de la tête et du cou [86-88]. A l'inverse, le phénotype immun-désert, caractérisé par une pénurie de cellules $\mathrm{T}$ dans le stroma et le parenchyme de la tumeur suggère l'absence d'immunité anti-tumorale préexistante et répond rarement aux traitements anti-PD-L1 / PD-1 [77,89,90]. Ainsi, l'évaluation de l'infiltrat immunitaire tumoral par imagerie médicale computationnelle semble être une piste prometteuse pour la recherche de nouveaux marqueurs d'efficacité de ces immunothérapies. Les données publiées à ce jour sont rares, mais plusieurs abstracts présentés lors de congrès internationaux témoignent de l'engouement de la recherche dans ce domaine. 
Orooji et al ont analysé les scanners de 17 patients atteints de cancers broncho-pulmonaires tumorale (évaluée par coloration à l'hématoxyline et à l'éosine) était considérée comme absente ou élevée [91]. Le training set était constitué de 12 patients, et 669 caractéristiques de texture et de formes étaient évaluées par analyse discriminante linéaire en vue de prédire l'infiltration lymphocytaire. Leur méthode a permis de classer correctement l'infiltration lymphocytaire de 4 des 5 patients du set de validation. Cependant, l'absence des détails de leur méthode d'apprentissage et le faible nombre de patients nécessitent de rester prudent dans l'interprétation des résultats [91]. Tang et al ont développé une signature radiomique dans les CBPNPC, prenant en compte la classification immunologique des tumeurs selon Teng et al [92,93]. Les patients étaient regroupés selon l'infiltration lymphocytaire tumorale (ILT) et l'expression de PD-L1 de leur tumeur, en 4 groupes : PDL1 ${ }^{\text {haut }} \operatorname{ILT}^{\text {haut }}(\mathrm{Gp} 1)$, PDL1 $^{\text {bas }}$ ILT $^{\text {bas }}$ (Gp2), PDL1 ${ }^{\text {haut }}$ ILT $^{\text {bas }}(\mathrm{Gp} 3)$, PDL1 ${ }^{\text {bas }}$ ILT $^{\text {haut }}(\mathrm{Gp} 4)$. A partir de scanners injectés, 490 index étaient extraits. Après sélection des variables l'apprentissage était réalisé sur 114 patients et validé sur une cohorte indépendante de 179 patients. La signature finale comportait 4 index robustes : moyenne globale, écart-type et uniformité des intensités, et homogénéité de la matrice de co-occurrence. Ce modèle était significativement associé à la survie en multivarié dans les deux cohortes, après ajustement sur le stade et le traitement adjuvant. Les auteurs poursuivent actuellement leur recherche en évaluant l'application du modèle pour sélectionner les patients à un traitement par radiothérapie combiné à de l'immunothérapie [92]. Dans une autre étude, Prawira et al ont analysé les scanners de 15 patients atteints de cancers de la tête et du cou, inclus prospectivement dans des essais de phase 1 d'immunothérapie (anti-PD-L1 : n=8, et anti-CTLA-4 : n=7). Quarante-deux lésions ont été délinéées manuellement, mais trente-trois ont été retenues pour analyse (lésions exclues pour données cliniques prématurées) [94]. Avec 20 lésions répondeuses, et 11 non-répondeuses sur le premier 
scanner d'évaluation, un modèle d'apprentissage par machine à vecteurs de support a été entrainé par validation croisée, et a montré une prédiction de 79\% (sensibilité 65,1\%, spécificité $88 \%, \mathrm{p}=0,029$ ) avec une aire sous la courbe ROC (Receiver Operating Characteristic) de 0,75 lorsque le modèle était entrainé sur le premier scanner d'évaluation, mais une prédiction de 70,8\% (sensibilité 37,9\%, spécificité 91.5\%, p =0.16) avec une aire sous la courbe ROC de 0,60 lorsqu'il était entrainé sur le scanner de référence pré-traitement [94]. Les index radiomiques utilisés ne sont pas décrits, et il n'y a pas de véritable cohorte de validation indépendante, mais le recrutement est toujours en cours afin d'améliorer leur modèle.

\section{Conclusion}

L'imagerie médicale computationnelle est une discipline particulièrement émergente en oncologie, bénéficiant de la masse importante de données d'imagerie réalisées pour le diagnostic et le suivi des patients. En France, en 2014, étaient ainsi réalisés 5 millions de scanners $(+24,8 \%$ par rapport à 2008), 3,6 millions d'IRM (+79\% par rapport à 2008$)$ et 0,8 millions d'examens de scintigraphie en général (+37,5\% par rapport à 2008) [95]. De plus, le partage d'examens d'imagerie annotés dans des portails publics, comme le The Cancer Imaging Archive (TCIA) [96] devrait permettre d'accélérer la recherche et d'améliorer les modèles d'apprentissage.

Cependant, même si les premiers résultats sont très encourageants, les challenges restent la standardisation des acquisitions d'imagerie [97], et la validation des signatures radiomiques à grande échelle avant de pouvoir mettre en place cette technique en pratique. Des recommandations pour la standardisation des pratiques, la validation et la communication des résultats, et le partage des données ont ainsi été émises afin de garantir une recherche 
scientifique de qualité et permettre une transposition efficace en clinique des biomarqueurs d'imagerie, et à terme, d'améliorer la prise en charge des patients [98]. 


\section{Bibliographie}

[1] Limkin EJ, Sun R, Dercle L, Zacharaki EI, Robert C, Reuzé S, et al. Promises and challenges for the implementation of computational medical imaging (radiomics) in oncology. Ann Oncol Off J Eur Soc Med Oncol 2017.

[2] Aerts HJWL, Velazquez ER, Leijenaar RTH, Parmar C, Grossmann P, Carvalho S, et al. Decoding tumour phenotype by noninvasive imaging using a quantitative radiomics approach. Nat Commun 2014;5:4006. doi:10.1038/ncomms5006.

[3] Gillies RJ, Kinahan PE, Hricak H. Radiomics: Images Are More than Pictures, They Are Data. Radiology 2015:151169. doi:10.1148/radiol.2015151169.

[4] O’Connor JPB, Rose CJ, Waterton JC, Carano RAD, Parker GJM, Jackson A. Imaging Intratumor Heterogeneity: Role in Therapy Response, Resistance, and Clinical Outcome. Clin Cancer Res 2015;21:249-57. doi:10.1158/1078-0432.CCR-14-0990.

[5] Reuzé S, Orlhac F, Chargari C, Nioche C, Limkin E, Riet F, et al. Prediction of cervical cancer recurrence using textural features extracted from 18 F-FDG PET images acquired with different scanners. Oncotarget 2017;5. doi:10.18632/oncotarget.17856.

[6] Sun R, Orlhac F, Robert C, Reuzé S, Schernberg A, Buvat I, et al. In Regard to Mattonen et al. Int J Radiat Oncol Biol Phys 2016;95:1544.

[7] Robert C, Long GV, Brady B, Dutriaux C, Maio M, Mortier L, et al. Nivolumab in Previously Untreated Melanoma without BRAF Mutation. N Engl J Med 2015;372:320-30. doi:10.1056/NEJMoa1412082.

[8] Weber JS, Kudchadkar RR, Yu B, Gallenstein D, Horak CE, Inzunza HD, et al. Safety, efficacy, and biomarkers of nivolumab with vaccine in ipilimumab-refractory or naive melanoma. J Clin Oncol Off J Am Soc Clin Oncol 2013;31:4311-8. doi:10.1200/JCO.2013.51.4802.

[9] Ansell SM. Hodgkin Lymphoma: Diagnosis and Treatment. Mayo Clin Proc 
2015;90:1574-83. doi:10.1016/j.mayocp.2015.07.005.

[10] Reck M, Rodríguez-Abreu D, Robinson AG, Hui R, Csőszi T, Fülöp A, et al. Pembrolizumab versus Chemotherapy for PD-L1-Positive Non-Small-Cell Lung Cancer. N Engl J Med 2016;375:1823-33. doi:10.1056/NEJMoa1606774.

[11] Motzer RJ, Escudier B, McDermott DF, George S, Hammers HJ, Srinivas S, et al. Nivolumab versus Everolimus in Advanced Renal-Cell Carcinoma. N Engl J Med 2015;373:1803-13. doi:10.1056/NEJMoa1510665.

[12] Chen DS, Mellman I. Elements of cancer immunity and the cancer-immune set point. Nature 2017;541:321-30. doi:10.1038/nature21349.

[13] Postow MA, Chesney J, Pavlick AC, Robert C, Grossmann K, McDermott D, et al. Nivolumab and ipilimumab versus ipilimumab in untreated melanoma. N Engl J Med 2015;372:2006-17. doi:10.1056/NEJMoa1414428.

[14] Hellmann MD, Rizvi NA, Goldman JW, Gettinger SN, Borghaei H, Brahmer JR, et al. Nivolumab plus ipilimumab as first-line treatment for advanced non-small-cell lung cancer (CheckMate 012): results of an open-label, phase 1, multicohort study. Lancet Oncol 2017;18:31-41. doi:10.1016/S1470-2045(16)30624-6.

[15] Bushberg JT, Boone JM. The essential physics of medical imaging. Lippincott Williams \& Wilkins; 2011.

[16] Galavis PE, Hollensen C, Jallow N, Paliwal B, Jeraj R. Variability of textural features in FDG PET images due to different acquisition modes and reconstruction parameters. Acta Oncol Stockh Swed 2010;49:1012-6. doi:10.3109/0284186X.2010.498437.

[17] Zhao B, Tan Y, Tsai W-Y, Qi J, Xie C, Lu L, et al. Reproducibility of radiomics for deciphering tumor phenotype with imaging. Sci Rep 2016;6:23428. doi:10.1038/srep23428.

[18] Balagurunathan Y, Kumar V, Gu Y, Kim J, Wang H, Liu Y, et al. Test-Retest Reproducibility Analysis of Lung CT Image Features. J Digit Imaging 2014;27:805-23. 
doi:10.1007/s10278-014-9716-X.

[19] Parmar C, Rios Velazquez E, Leijenaar R, Jermoumi M, Carvalho S, Mak RH, et al. Robust Radiomics Feature Quantification Using Semiautomatic Volumetric Segmentation. PLoS ONE 2014;9. doi:10.1371/journal.pone.0102107.

[20] Rios Velazquez E, Aerts HJWL, Gu Y, Goldgof DB, De Ruysscher D, Dekker A, et al. A semiautomatic CT-based ensemble segmentation of lung tumors: comparison with oncologists' delineations and with the surgical specimen. Radiother Oncol J Eur Soc Ther Radiol Oncol 2012;105:167-73. doi:10.1016/j.radonc.2012.09.023.

[21] Orlhac F, Soussan M, Maisonobe J-A, Garcia CA, Vanderlinden B, Buvat I. Tumor texture analysis in 18F-FDG PET: relationships between texture parameters, histogram indices, standardized uptake values, metabolic volumes, and total lesion glycolysis. J Nucl Med Off Publ Soc Nucl Med 2014;55:414-22. doi:10.2967/jnumed.113.129858.

[22] Velazquez ER, Parmar C, Jermoumi M, Mak RH, van Baardwijk A, Fennessy FM, et al. Volumetric CT-based segmentation of NSCLC using 3D-Slicer. Sci Rep 2013;3. doi:10.1038/srep03529.

[23] Gorthi S, Bach Cuadra M, Thiran J-P. Exporting Contours to DICOM-RT Structure Set. Insight J 2009.

[24] FormatAnalyze - MRC CBU Imaging Wiki n.d. http://imaging.mrccbu.cam.ac.uk/imaging/FormatAnalyze (accessed January 16, 2017).

[25] mjenkinson. NIfTI-1 Data Format - Neuroimaging Informatics Technology Initiative n.d. http://nifti.nimh.nih.gov/nifti-1 (accessed September 5, 2016).

[26] Wells WM. Efficient Synthesis of Gaussian Filters by Cascaded Uniform Filters. IEEE Trans Pattern Anal Mach Intell 1986;PAMI-8:234-9. doi:10.1109/TPAMI.1986.4767776.

[27] Leijenaar RTH, Nalbantov G, Carvalho S, van Elmpt WJC, Troost EGC, Boellaard R, et al. The effect of SUV discretization in quantitative FDG-PET Radiomics: the need for 
standardized methodology in tumor texture analysis. Sci Rep 2015;5. doi:10.1038/srep11075.

[28] Orlhac F, Soussan M, Chouahnia K, Martinod E, Buvat I. 18F-FDG PET-Derived Textural Indices Reflect Tissue-Specific Uptake Pattern in Non-Small Cell Lung Cancer. PloS One 2015;10:e0145063. doi:10.1371/journal.pone.0145063.

[29] Brooks FJ, Grigsby PW. The effect of small tumor volumes on studies of intratumoral heterogeneity of tracer uptake. J Nucl Med Off Publ Soc Nucl Med 2014;55:37-42. doi:10.2967/jnumed.112.116715.

[30] van Velden FHP, Kramer GM, Frings V, Nissen IA, Mulder ER, de Langen AJ, et al. Repeatability of Radiomic Features in Non-Small-Cell Lung Cancer [(18)F]FDG-PET/CT Studies: Impact of Reconstruction and Delineation. Mol Imaging Biol MIB Off Publ Acad Mol Imaging 2016. doi:10.1007/s11307-016-0940-2.

[31] El Naqa I, Grigsby PW, Apte A, Kidd E, Donnelly E, Khullar D, et al. Exploring feature-based approaches in PET images for predicting cancer treatment outcomes. Pattern Recognit 2009;42:1162-71. doi:10.1016/j.patcog.2008.08.011.

[32] Haralick RM, Shanmugam K, Dinstein I. Textural Features for Image Classification. IEEE Trans Syst Man Cybern 1973;SMC-3:610-21. doi:10.1109/TSMC.1973.4309314.

[33] Galloway MM. Texture analysis using gray level run lengths. Comput Graph Image Process 1975;4:172-9. doi:10.1016/S0146-664X(75)80008-6.

[34] Thibault G, Angulo J, Meyer F. Advanced Statistical Matrices for Texture Characterization: Application to Cell Classification. IEEE Trans Biomed Eng 2014;61:630-7. doi:10.1109/TBME.2013.2284600.

[35] Sun C, Wee WG. Neighboring gray level dependence matrix for texture classification. Comput Vis Graph Image Process 1983;23:341-52. doi:10.1016/0734-189X(83)90032-4.

[36] Narang S, Lehrer M, Yang D, Lee J, Rao A. Radiomics in glioblastoma: current status, challenges and potential opportunities. Transl Cancer Res 2016;5:383-97. doi:10.21037/8806. 
[37] Zheng Y, Englander S, Baloch S, Zacharaki EI, Fan Y, Schnall MD, et al. STEP: Spatiotemporal enhancement pattern for MR-based breast tumor diagnosis. Med Phys 2009;36:3192-204. doi:10.1118/1.3151811.

[38] Coroller TP, Grossmann P, Hou Y, Rios Velazquez E, Leijenaar RTH, Hermann G, et al. CT-based radiomic signature predicts distant metastasis in lung adenocarcinoma. Radiother Oncol J Eur Soc Ther Radiol Oncol 2015;114:345-50. doi:10.1016/j.radonc.2015.02.015.

[39] Kickingereder P, Götz M, Muschelli J, Wick A, Neuberger U, Shinohara RT, et al. Large-scale Radiomic Profiling of Recurrent Glioblastoma Identifies an Imaging Predictor for Stratifying Anti-Angiogenic Treatment Response. Clin Cancer Res Off J Am Assoc Cancer Res 2016;22:5765-71. doi:10.1158/1078-0432.CCR-16-0702.

[40] Bellman R. Adaptive control processes: a guided tour. Princeton: Princeton university press; 1961.

[41] Clarke R, Ressom HW, Wang A, Xuan J, Liu MC, Gehan EA, et al. The properties of high-dimensional data spaces: implications for exploring gene and protein expression data. Nat Rev Cancer 2008;8:37-49. doi:10.1038/nrc2294.

[42] Ferte C, Trister AD, Huang E, Bot BM, Guinney J, Commo F, et al. Impact of Bioinformatic Procedures in the Development and Translation of High-Throughput Molecular Classifiers in Oncology. Clin Cancer Res 2013;19:4315-25. doi:10.1158/1078-0432.CCR-123937.

[43] Holm S. A Simple Sequentially Rejective Multiple Test Procedure. Scand J Stat $1979 ; 6: 65-70$.

[44] Y HY and B. More powerful procedures for multiple significance testing. - PubMed NCBI n.d. https://vpn.igr.fr/pubmed/,DanaInfo=www.ncbi.nlm.nih.gov,SSL+2218183 (accessed January 13, 2017). 
[45] Parmar C, Grossmann P, Bussink J, Lambin P, Aerts HJWL. Machine Learning methods for Quantitative Radiomic Biomarkers. Sci Rep 2015;5. doi:10.1038/srep13087.

[46] Armato SG, Meyer CR, Mcnitt-Gray MF, McLennan G, Reeves AP, Croft BY, et al. The Reference Image Database to Evaluate Response to therapy in lung cancer (RIDER) project: a resource for the development of change-analysis software. Clin Pharmacol Ther 2008;84:448-56. doi:10.1038/clpt.2008.161.

[47] Peng H, Long F, Ding C. Feature selection based on mutual information: criteria of max-dependency, max-relevance, and min-redundancy. IEEE Trans Pattern Anal Mach Intell 2005;27:1226-38. doi:10.1109/TPAMI.2005.159.

[48] Kononenko I. Estimating attributes: Analysis and extensions of RELIEF. Mach. Learn. ECML-94, Springer, Berlin, Heidelberg; 1994, p. 171-82. doi:10.1007/3-540-57868-4_57.

[49] Kohavi R, John GH. Wrappers for feature subset selection. Artif Intell 1997;97:273324. doi:10.1016/S0004-3702(97)00043-X.

[50] Zou H, Hastie T. Regularization and Variable Selection via the Elastic Net. J R Stat Soc Ser B Stat Methodol 2005;67:301-20.

[51] Fisher RA. THE USE OF MULTIPLE MEASUREMENTS IN TAXONOMIC PROBLEMS. Ann Eugen 1936;7:179-88. doi:10.1111/j.1469-1809.1936.tb02137.x.

[52] Tenenbaum JB, de Silva V, Langford JC. A global geometric framework for nonlinear dimensionality reduction. Science 2000;290:2319-23. doi:10.1126/science.290.5500.2319.

[53] Roweis ST, Saul LK. Nonlinear dimensionality reduction by locally linear embedding. Science 2000;290:2323-6. doi:10.1126/science.290.5500.2323.

[54] Hinton GE. Reducing the Dimensionality of Data with Neural Networks. Science 2006;313:504-7. doi:10.1126/science.1127647.

[55] Handbook of Biomedical Imaging - Methodologies and Clinical | Nikos Paragios | Springer. n.d. 
[56] Shao J. Linear Model Selection by Cross-validation. J Am Stat Assoc 1993;88:48694. doi:10.1080/01621459.1993.10476299.

[57] Kumar D, Shafiee MJ, Chung AG, Khalvati F, Haider MA, Wong A. Discovery Radiomics for Computed Tomography Cancer Detection. ArXiv150900117 Cs 2015.

[58] Hawkins S, Wang H, Liu Y, Garcia A, Stringfield O, Krewer H, et al. Predicting Malignant Nodules from Screening CT Scans. J Thorac Oncol 2016. doi:10.1016/j.jtho.2016.07.002.

[59] Shen W, Zhou M, Yang F, Yang C, Tian J. Multi-scale Convolutional Neural Networks for Lung Nodule Classification. In: Ourselin S, Alexander DC, Westin C-F, Cardoso MJ, editors. Inf. Process. Med. Imaging, vol. 9123, Cham: Springer International Publishing; 2015, p. 588-99.

[60] Zacharaki EI, Morita N, Bhatt P, O’Rourke DM, Melhem ER, Davatzikos C. Survival Analysis of Patients with High-Grade Gliomas Based on Data Mining of Imaging Variables. AJNR Am J Neuroradiol 2012;33:1065. doi:10.3174/ajnr.A2939.

[61] Zacharaki EI, Kanas VG, Davatzikos C. Investigating machine learning techniques for MRI-based classification of brain neoplasms. Int J Comput Assist Radiol Surg 2011;6:821-8. doi:10.1007/s11548-011-0559-3.

[62] al LY et. Radiological Image traits Predictive of Cancer Status in Pulmonary Nodules. $\begin{array}{llll}\text { PubMed } & - & \text { NCBI } & \text { n.d. }\end{array}$ https://vpn.igr.fr/pubmed/,DanaInfo=www.ncbi.nlm.nih.gov,SSL+27663588 ～（accessed December 2, 2016).

[63] Zacharaki EI, Wang S, Chawla S, Soo Yoo D, Wolf R, Melhem ER, et al. Classification of brain tumor type and grade using MRI texture and shape in a machine learning scheme. Magn Reson Med 2009;62:1609-18. doi:10.1002/mrm.22147.

[64] Wang J, Liu X, Dong D, Song J, Xu M, Zang Y, et al. Prediction of malignant and 
benign of lung tumor using a quantitative radiomic method. 2016 38th Annu. Int. Conf. IEEE

Eng. Med. Biol. Soc. EMBC, 2016, p. 1272-5. doi:10.1109/EMBC.2016.7590938.

[65] Nie K, Shi L, Chen Q, Hu X, Jabbour S, Yue N, et al. Rectal Cancer: Assessment of Neoadjuvant Chemo-Radiation Outcome Based on Radiomics of Multi-Parametric MRI. Clin Cancer Res 2016. doi:10.1158/1078-0432.CCR-15-2997.

[66] Hastie T, Tibshirani R, Friedman J. Unsupervised learning. Elem. Stat. Learn., Springer; 2009, p. 485-585.

[67] LeCun Y, Bengio Y, Hinton G. Deep learning. Nature 2015;521:436-44. doi:10.1038/nature14539.

[68] Zhang Q, Xiao Y, Dai W, Suo J, Wang C, Shi J, et al. Deep learning based classification of breast tumors with shear-wave elastography. Ultrasonics 2016;72:150-7. doi:10.1016/j.ultras.2016.08.004.

[69] Cheng J-Z, Ni D, Chou Y-H, Qin J, Tiu C-M, Chang Y-C, et al. Computer-Aided Diagnosis with Deep Learning Architecture: Applications to Breast Lesions in US Images and Pulmonary Nodules in CT Scans. Sci Rep 2016;6:24454. doi:10.1038/srep24454.

[70] Sohn K, Zhou G, Lee C, Lee H. Learning and Selecting Features Jointly with Pointwise Gated Boltzmann Machines., 2013, p. 217-25.

[71] Carneiro G, Oakden-Rayner L, Bradley AP, Nascimento J, Palmer L. Automated 5year Mortality Prediction using Deep Learning and Radiomics Features from Chest Computed Tomography 2016.

[72] Hanahan D, Weinberg RA. Hallmarks of Cancer: The Next Generation. Cell 2011;144:646-74. doi:10.1016/j.cell.2011.02.013.

[73] Couzin-Frankel J. Breakthrough of the year 2013. Cancer immunotherapy. Science 2013;342:1432-3. doi:10.1126/science.342.6165.1432.

[74] Wolchok JD, Kluger H, Callahan MK, Postow MA, Rizvi NA, Lesokhin AM, et al. 
Nivolumab plus Ipilimumab in Advanced Melanoma. N Engl J Med 2013;369:122-33. doi:10.1056/NEJMoa1302369.

[75] Robert C, Schachter J, Long GV, Arance A, Grob JJ, Mortier L, et al. Pembrolizumab versus Ipilimumab in Advanced Melanoma. N Engl J Med 2015;372:2521-32. doi:10.1056/NEJMoa1503093.

[76] Zou W, Wolchok JD, Chen L. PD-L1 (B7-H1) and PD-1 pathway blockade for cancer therapy: Mechanisms, response biomarkers, and combinations. Sci Transl Med 2016;8:328rv4. doi:10.1126/scitranslmed.aad7118.

[77] Herbst RS, Soria J-C, Kowanetz M, Fine GD, Hamid O, Gordon MS, et al. Predictive correlates of response to the anti-PD-L1 antibody MPDL3280A in cancer patients. Nature 2014;515:563-7.

[78] Kim JM, Chen DS. Immune escape to PD-L1/PD-1 blockade: seven steps to success (or failure). Ann Oncol 2016;27:1492-504. doi:10.1093/annonc/mdw217.

[79] Hugo W, Zaretsky JM, Sun L, Song C, Moreno BH, Hu-Lieskovan S, et al. Genomic and Transcriptomic Features of Response to Anti-PD-1 Therapy in Metastatic Melanoma. Cell 2016;165:35-44. doi:10.1016/j.cell.2016.02.065.

[80] Ribas A, Shin DS, Zaretsky J, Frederiksen J, Cornish A, Avramis E, et al. PD-1 Blockade Expands Intratumoral Memory T Cells. Cancer Immunol Res 2016;4:194-203. doi:10.1158/2326-6066.CIR-15-0210.

[81] Tumeh PC, Harview CL, Yearley JH, Shintaku IP, Taylor EJM, Robert L, et al. PD-1 blockade induces responses by inhibiting adaptive immune resistance. Nature 2014;515:56871. doi:10.1038/nature13954.

[82] Rosenberg JE, Hoffman-Censits J, Powles T, van der Heijden MS, Balar AV, Necchi A, et al. Atezolizumab in patients with locally advanced and metastatic urothelial carcinoma who have progressed following treatment with platinum-based chemotherapy: a single-arm, 
multicentre, phase 2 trial. Lancet Lond Engl 2016;387:1909-20. doi:10.1016/S01406736(16)00561-4.

[83] Clemente CG, Mihm MC, Bufalino R, Zurrida S, Collini P, Cascinelli N. Prognostic value of tumor infiltrating lymphocytes in the vertical growth phase of primary cutaneous melanoma. Cancer 1996;77:1303-10. doi:10.1002/(SICI)10970142(19960401)77:7<1303::AID-CNCR12>3.0.CO;2-5.

[84] Sato E, Olson SH, Ahn J, Bundy B, Nishikawa H, Qian F, et al. Intraepithelial CD8+ tumor-infiltrating lymphocytes and a high CD8+/regulatory $\mathrm{T}$ cell ratio are associated with favorable prognosis in ovarian cancer. Proc Natl Acad Sci U S A 2005;102:18538-43. doi:10.1073/pnas.0509182102.

[85] Pagès F, Berger A, Camus M, Sanchez-Cabo F, Costes A, Molidor R, et al. Effector memory $\mathrm{T}$ cells, early metastasis, and survival in colorectal cancer. $\mathrm{N}$ Engl $\mathrm{J}$ Med 2005;353:2654-66. doi:10.1056/NEJMoa051424.

[86] Ogino T, Shigyo H, Ishii H, Katayama A, Miyokawa N, Harabuchi Y, et al. HLA class I antigen down-regulation in primary laryngeal squamous cell carcinoma lesions as a poor prognostic marker. Cancer Res 2006;66:9281-9. doi:10.1158/0008-5472.CAN-06-0488.

[87] Balermpas P, Michel Y, Wagenblast J, Seitz O, Weiss C, Rödel F, et al. Tumourinfiltrating lymphocytes predict response to definitive chemoradiotherapy in head and neck cancer. Br J Cancer 2014;110:501-9. doi:10.1038/bjc.2013.640.

[88] Wansom D, Light E, Thomas D, Worden F, Prince M, Urba S, et al. INFILTRATING LYMPHOCYTES AND HUMAN PAPILLOMAVIRUS-16 ASSOCIATED OROPHARYNX CANCER. The Laryngoscope 2012;122:121-7. doi:10.1002/lary.22133.

[89] Gajewski TF, Woo S-R, Zha Y, Spaapen R, Zheng Y, Corrales L, et al. Cancer immunotherapy strategies based on overcoming barriers within the tumor microenvironment. Curr Opin Immunol 2013;25:268-76. doi:10.1016/j.coi.2013.02.009. 
[90] Hegde PS, Karanikas V, Evers S. The Where, the When, and the How of Immune Monitoring for Cancer Immunotherapies in the Era of Checkpoint Inhibition. Clin Cancer Res Off J Am Assoc Cancer Res 2016;22:1865-74. doi:10.1158/1078-0432.CCR-15-1507.

[91] Orooji M, Rakshit S, Beig N, Madabhushi A, Velcheti V. Computerized textural analysis of lung CT to enable quantification of tumor infiltrating lymphocytes in NSCLC. $\mathbf{J}$ Clin Oncol 2016;34:11584-11584. doi:10.1200/JCO.2016.34.15_suppl.11584.

[92] Tang C, Amer A, Hobbs B, Li X, Behrens C, Para Cuentas E, et al. Pathology-Based Non-Small Cell Lung Cancer Radiomics Signature Describing the Local Tumor Immune Environment: Discovery and Validation. Int J Radiat Oncol 2016;96:S42-3. doi:10.1016/j.ijrobp.2016.06.114.

[93] Teng MWL, Ngiow SF, Ribas A, Smyth MJ. Classifying Cancers Based on T-cell Infiltration and PD-L1. Cancer Res 2015;75:2139-45. doi:10.1158/0008-5472.CAN-15-0255. [94] Prawira A, Dufort P, Halankar J, Paravasthu DM, Hansen A, Spreafico A, et al. Development of a predictive radiomics signature for response to immune checkpoint inhibitors (ICIs) in patients with recurrent or metastatic squamous cell carcinoma of the head and neck (RM-SCCHN). Ann Oncol 2016;27. doi:10.1093/annonc/mdw376.08.

[95] HCAAM - document annexé au rapport « innovation et système de santé ». Document 13 : les équipements lourds d'imagerie. 2016. http://www.securitesociale.fr/IMG/pdf/document_13_-_les_equipements_lourds_d_imagerie.pdf (accessed June $8,2017)$.

[96] Clark K, Vendt B, Smith K, Freymann J, Kirby J, Koppel P, et al. The Cancer Imaging Archive (TCIA): Maintaining and Operating a Public Information Repository. J Digit Imaging 2013;26:1045-57. doi:10.1007/s10278-013-9622-7.

[97] Mackin D, Fave X, Zhang L, Fried D, Yang J, Taylor B, et al. Measuring Computed Tomography Scanner Variability of Radiomics Features. Invest Radiol 2015;50:757-65. 
doi:10.1097/RLI.0000000000000180.

[98] O’Connor JPB, Aboagye EO, Adams JE, Aerts HJWL, Barrington SF, Beer AJ, et al. Imaging biomarker roadmap for cancer studies. Nat Rev Clin Oncol 2017;14:169-86. doi:10.1038/nrclinonc.2016.162. 


\section{FIGURES}

Figure 1 : Processus de l'analyse d'imagerie computationnelle (radiomics) : acquisition de l'image et segmentation, extraction des caractéristiques d'imagerie ou index, et apprentissage statistique. L'analyse des données intègre les données de la clinique, de l'anatomo-pathologie, de la génomique en vue d'une médecine de précision.

Figure 1: The computational imaging (radiomics) pipeline, showing the major steps: image acquisition and segmentation, feature extraction, and statistical learning. Radiomics data are meant to be integrated and analyzed with clinical, pathologic and -omics data to improve precision medicine.

Figure reproduite avec l'autorisation de Limkin E et al [1] 
Figure 1

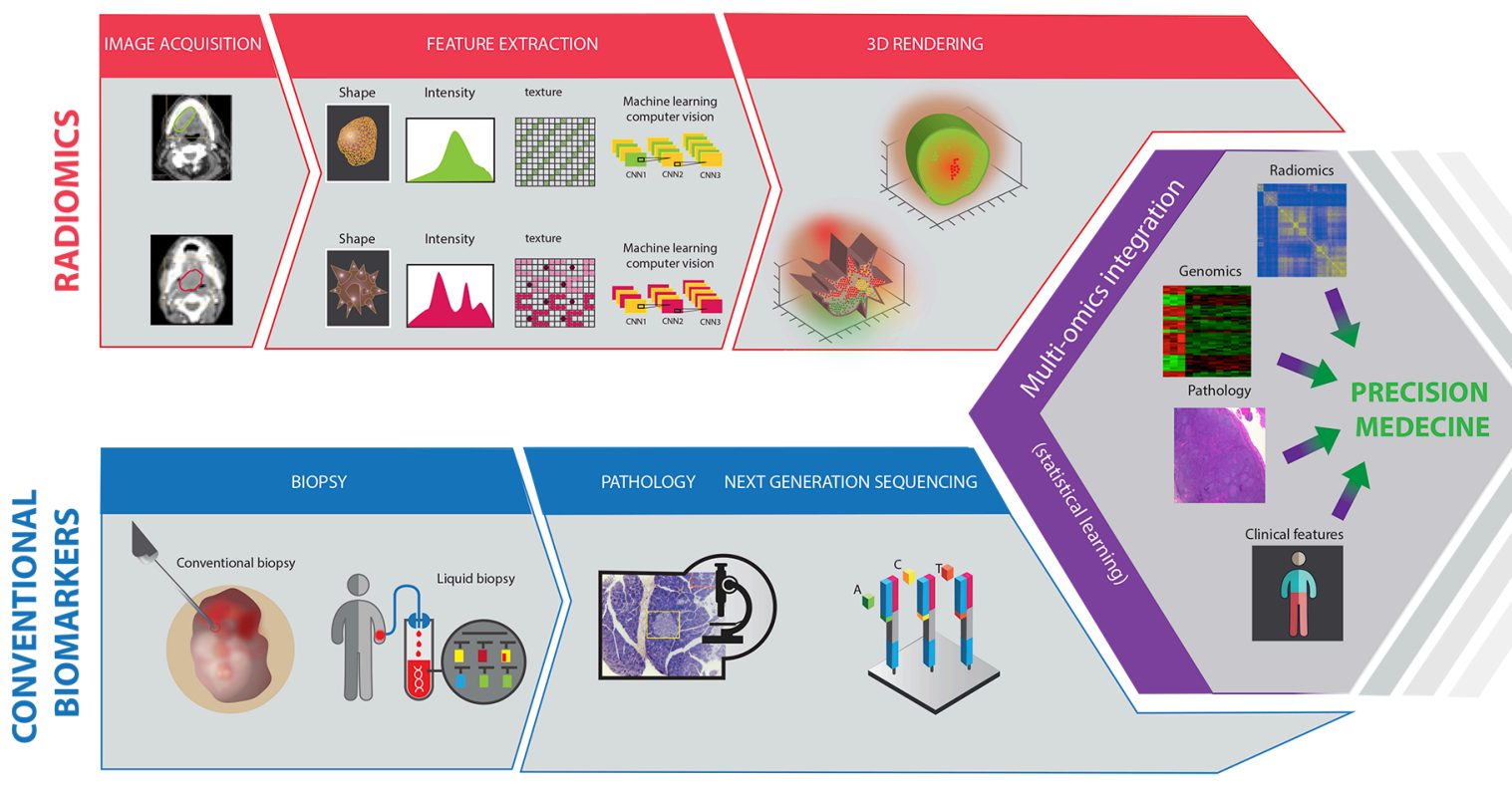

